\title{
Internacionalización e interlocución entre la educación básica y superior: el problema de la formación docente
}

\author{
Internacionalização e a interlocução entre o ensino básico e superior: o \\ problema da formação docente
}

\begin{abstract}
Internationalization and the dialogue between basic higher education: the problem of teacher training
\end{abstract}

\section{Patricia Viera-Duarte ${ }^{1}$}

Universidad de la República, Professora no Centro de Políticas Educativas

Resumen: En el escenario latinoamericano, determinado por las tendencias internacionales del mundo globalizado - por un lado - y por las demandas de las políticas educativas nacionales -por otro- comienzan a hacerse visibles ciertas tensiones que dificultan una comprensión sistémica de la educación en el contexto Regional y dentro de los sistemas de enseñanza de cada país. Las investigaciones que sustentan estas reflexiones, tienen por objetivo instalar el tema en una inter/relación entre: políticas educativas nacionales - posiblemente determinadas por parámetros internacionales $-\mathrm{y}$ sus realidades institucionales locales. A través de exploración de documentos de organismos multilaterales; de entrevistas a los actores nacionales encargados del diseño y desarrollo de las políticas educativas; así como de varios focus groups con gestores y docentes locales, el problema se analizó en términos de "relación" entre políticas internacionales y políticas educativas en territorio. Específicamente, en este artículo se abordan las interlocuciones entre las demandas y desafíos de la Educación Básica - de cada país - con las innovaciones de la Formación Docente instalada dentro de una Educación Superior cada vez más internacionalizada. Se trata de una reflexión a partir de una serie de estudios de corte cualitativo, dentro del contexto de la hermenéutica, que concluye con algunas recomendaciones para re/articular los diálogos regionales/nacionales/sub nacionales, en sus distintos niveles.

Palabras clave: Políticas educativas. Internacionalización de la Educación Superior. Educación Básica. Formación Docente.

\footnotetext{
1 Doctora en Educación con énfasis en Políticas Educativas por la Universidad de La Empresa; Máster en Educación con énfasis en investigación, enseñanza y aprendizajes por la Universidad ORT.
} 
Abstract: In the Latin American scene, determined by international trends of the globalized world - on one hand, and the demands of national education policies on the other, certain tensions become visible whereas hinder a systemic understanding of education in the regional context and within education systems in each country. The research supporting these reflections, it was used: documentary analysis, interviews, focus groups; and set up the theme in an inter / relationship: national education policies, which are determined by international parameters and local institutional realities. The problem arises in terms of the relationship between international policies patterned by multilateral agencies and educational policies in the territory. Specifically, in this article the interlocutions between the demands and challenges of Basic Education in each country with the innovations of Teacher Education installed within an increasingly internationalized higher education are addressed. This is a series of qualitative studies within the context of hermeneutics, which concludes with some recommendations for re / articulate national regional / national / sub relationships at various levels.

Keywords: Education policies. Internationalization of Higher Education. Basic Education. Teacher Training.

Resumo: No cenário latino-americano, determinado pelas tendências internacionais do mundo globalizado - por um lado - e pelas exigências das políticas nacionais de educação por outro, começam a se tornar visíveis certas tensões que dificultam a compreensão sistêmica da educação no contexto regional e dentro dos sistemas de ensino em cada país. As pesquisas que sustentam essas reflexões têm por objetivo colocar o tema nas inter/relações entre as políticas nacionais de educação-possivelmente determinadas pelos parâmetros internacionais - e as realidades institucionais locais. A partir de explorações de documentos dos organismos multilaterais, entrevistas aos atores nacionais encarregados do desenho e desenvolvimento das politicas educativas e de realização de grupos focais com gestores e professores locais, o problema foi analisado em termos da relação entre as políticas internacionais e as politicas educacionais no território. Neste artigo, serão abordadas, com mais ênfase, as interlocuções entre as demandas e desafios da educação básica, em cada país, com as inovações da formação de professores instalados dentro de um Ensino Superior cada vez mais internacionalizado. Este trabalho é parte de uma série de estudos qualitativos no contexto da hermenêutica, que conclui com algumas recomendações para (re) articular os diálogos regionais, nacionais e subnacionais, nos seus diversos níveis.

Palavras-chave: Políticas educacionais. Internacionalização da Educação Superior. Educação Básica. Formação de professores. 


\section{INTRODUCCIÓN}

Con el presente artículo, se pretende realizar un aporte a la reflexión acerca de las interlocuciones necesarias entre la Formación Docente de Nivel Superior y la Educación Básica en un escenario tensionado por determinaciones de un mundo globalizado y por las - aún vigentes - demandas de los sistemas educativos nacionales. La formulación de políticas regionales parecería provocar una serie de desafíos para la Educación Básica y sus instituciones en contexto; dicho de otra forma, se desatan una serie de interferencias de las políticas públicas internacionales en las realidades nacionales y locales.

El objetivo de este trabajo es plantear la producción del conocimiento desde la comprensión sistémica de la Educación en el contexto regional. Los datos se desprenden de una serie de estudios comparados de los sistemas nacionales de enseñanza y - a su vez - una comparación entre los diversos subsistemas de la Educación dentro de cada país (VIERA, 2005, 2014, 2016). Este problema complejo - por su naturaleza misma - se instala dentro de la relación existente entre las políticas educativas determinadas, muchas veces, por parámetros internacionales y las realidades institucionales locales en contextos particulares; por tanto el problema se encuentra ubicado justamente en la intersección de varios planos: las políticas pautadas por organismos multilaterales, los sistemas nacionales de enseñanza y la implementación de las mismas en territorio.

No se tematiza, aquí, las causas de un fenómeno sino que se procura evitar sesgos causalísticos abordando el tema desde el paradigma de la complejidad (MORIN, 1998), o sea en términos de encuentro/desencuentro - en una relación dialógica hologramática y recursiva - entre organismos multilaterales, las políticas de los sistemas nacionales de Educación y las culturas locales en contexto, abordando el conocimiento desde la perspectiva de los sujetos y de lo subjetivo como lo plantea Ibáñez (1994).

En este artículo, se hará énfasis en el fenómeno de la relación Formación Docente -instalada dentro de una Educación Superior cada vez más internacionalizada - y las demandas de la Educación Básica, de cada país, enmarcadas en un sistema educativo nacional. Por lo antedicho, no se trata de estudiar efectos de un plano sobre el otro, por el contrario, se ha buscado estudiar las reacciones en las que serían posibles - también- retroacciones de "lo local" hacia "lo nacional" (VIERA, 2013); 
y de la misma manera posibilidades de retroacciones desde los sistemas nacionales hacia las políticas internacionales referidas a la Educación.

En el primer segmento del artículo se realiza una breve comparación entre el origen de las universidades y los mandatos fundacionales de los sistemas educativos, desde una perspectiva histórica, con el objetivo de comprender la complejidad de la coexistencia actual de estas instituciones con sellos fundacionales tan diferentes; pero ambas fundadas con la lógica propia de la modernidad que - además - en el siglo XXI está en plena crisis.

En este contexto de inflexión de racionalidades se plantean el problema de la formación docente que presenta diferencias de tradiciones dentro de Uruguay (VIERA, 2014), en Argentina y Brasil; pero que - a su vez - comparten los efectos de la internacionalización de la Educación Superior.

Para abarcar la complejidad del tema, se hace inevitable rever los supuestos epistemológicos tradicionales de los estudios comparados y de las políticas educativas. Por lo tanto se problematizan también las metodologías utilizadas en este campo disciplinar y la necesidad de un cambio de paradigma para lograr investigaciones más adecuadas a la naturaleza de este objeto de estudio.

Finalmente, el artículo, entrega algunas consideraciones finales, para nada exhaustivas, que derivan de la revisión de la literatura y de investigaciones anteriores. Se dejan abiertas nuevas cuestiones sobre el estudio de fenómenos sociales - políticos - educativos interdisciplinarios y sistémicos para futuros abordajes.

\section{UNIVERSIDADES Y EDUCACIÓN BÁSICA: TRADICIONES Y DESAFÍOS}

La historia de las universidades es distinta al origen y desarrollo de los sistemas nacionales de enseñanza básica. Las primeras, en su génesis fueron internacionales en el abordaje del conocimiento, mientras que los sistemas de educación básica surgen funcionales a los nuevos Estados republicanos - luego de la revolución Francesa - con el mandato de formar ciudadanos para las nuevas repúblicas; y así consolidar las nuevas identidades nacionales.

De acuerdo con Tello (2015), la modernidad hasta los siglos XIX y XX no exigía un diálogo entre los niveles básicos de la "educación para todos" y las universidades destinadas a las elites intelectuales. Sin embargo, para Rama Vitale 
(2016) - desde el modelo Napoleónico de 1806, el modelo de Humbolt de 1810, el modelo soviético de 1918 y el modelo de Córdoba de 1918 - las universidades se basan en políticas nacionales de investigación, enseñanza, acceso estudiantil y, de esta manera, comienzan a quedar encerradas en sus fronteras nacionales. Para Rama Vitale (2016), en Latinoamérica esa dependencia se reafirmó desde que Bolívar estatizó las universidades religiosas que posteriormente, con la reforma de Córdoba, se consolidó en el modelo nacional de autonomía y cogobierno.

Ahora, bien, en el siglo XXI, hay vientos de cambio y regresan las tendencias a los enfoque de internacionalización de las universidades, aunque en nuevos formatos.

Hoy estamos ante una nueva fase marcada por la transición hacia instituciones sin fronteras, con pertinencias globales, con estándares de calidad internacionales y con niveles de inserción en redes globales de investigación e intercambio lo cual deriva también en tensiones políticas y académicas frente a sus más claras manifestaciones como los Tratados de Libre Comercio, los acuerdos de integración en el MERCOSUR y las negociaciones de la OMC y de la OMPI [...] Sin embargo, es posible visualizar la tendencia a un lento camino que genera una cierta desnacionalización de la educación superior, y los gobiernos una cierta pérdida de su capacidad de fiscalización y control, que inclusive va conformando a la educación como bien público internacional tensionando el sistema político hacia nuevas formas de protección del conocimiento local antes inéditas. (RAMA VITALE, 2016, p. 202, subrayado nuestro).

Los rápidos y permanentes cambios de este siglo impactaron en las universidades, incluso antes; desde la posguerra del siglo XX se habían generado cambios profundos en el concepto de universidad: el desarrollo de la tecnología, la transición de la sociedad industrial a la de la información y ahora del conocimiento, provoca masificación en su acceso y se comienza a discutir el fenómeno de la democratización de la Educación Superior.

Los efectos de la internacionalización de una sociedad globalizada generan posibilidades de movilidad - estudiantil y docente - y disponibilidad de comunicación virtual, asociadas a la necesidad de apertura a las diversidades y a las demandas de una educación supranacional de fronteras difusas (PÉREZ LINDO, 2016). En el mismo sentido, en su conferencia titulada La misión de la universidad en el Siglo XXI, Corrales Ayala (2007) definía nuevos desafíos para la universidad en estos tiempos, 
entre ellos el desarrollo de todas las potencialidades de las personas entendidas en sus dimensiones bio-psico-sociales.

Para abordar la educación integral de las persona - en su conjunto - debe proponerse un cambio de pensamiento, por lo tanto, un cambio en los fines de la Educación Superior, que necesita crear vías de comunicación con la sociedad y con los demás agentes educativos. Desde estos planteos, Corrales Ayala (2007), afirmaba que se requiere que las universidades cambien su orientación predominantemente racional y asuman el desarrollo integral de las personas que están insertas en una sociedad. Todo esto, implica necesariamente pensar las políticas educativas de los sistemas de enseñanza en conjunto con todas sus modalidades y niveles desde una perspectiva dialógica.

En este panorama, de acuerdo con Fazio (2014), se hace imprescindible trabajar en redes; coloca como ejemplo la Red de Internacionalización y Movilidades Académicas y Científicas (RIMAC); en este caso se explicita que los objetivos de la red son los siguientes:

Generar un espacio de reflexión sobre marcos teóricos y normativos cuyos ajustes no solo requieren ser atendidos en el marco de la integración educativa superior, sino también representan aspectos o variables a tener en cuenta como viabilizadores u obstaculizadores de las estrategias que se emprendan en prospectiva [...] Identificar los retos de la integración educativa regional e internacional en Educación Superior, relacionados con la realidad "nacional" de los países de América Latina. (FAZIO, 2014, p. 1).

Se enfatiza, así la relevancia de investigar desde una visión integral y contextualizada del problema a fin de generar insumos para la toma de decisiones. La misma autora, en su Conferencia Internacional sobre currículum y Educación Comparada concluía que el desafío consiste en transformar el currículum desde una perspectiva comparada en el marco de los actuales lineamientos regionales e internacionales en armonía con la identidad y trayectoria nacional y que para lograrlo es necesario involucrar a todos los sujetos miembros de los sistemas educativos (FAZIO, 2015). 


\section{POLÍTICAS EDUCATIVAS NACIONALES PARA LA EDUCACIÓN BÁSICA Y LA FORMACIÓN DE SUS DOCENTES}

Las políticas públicas nacionales han estado abocadas a la universalización de la Educación Básica y con ello a mejorar la formación del magisterio. En este tema, existen diferentes tendencias: la formación normalista de modelo francés y la formación universitaria a través de carreras de grado para esta profesión; a partir de esta situación se generan encuentros y desencuentros, des/articulaciones y acciones tendientes a la interlocución necesaria entre ambos niveles de educación: Formación de Docentes y Enseñanza Fundamental.

Esta brecha se presenta de formas muy distintas en cada país de la región. De la revisión de la literatura, se desprende que, en el caso de Uruguay, existe una impronta - en esta materia - que fortalece las fragmentaciones entre los distintos niveles del Sistema Educativo; sin embargo la Educación Primaria en este país se organizó-desde fines del siglo XIX - planificada en forma conjunta con la formación del Magisterio para el nivel Primario. Las primeras Reformas Educativas en Argentina y en Uruguay - han abordado los cambios articulando la universalización del nivel básico con la formación de sus docentes y se abocaron a organizar el Sistema Nacional de Educación para universalizar la escuela primaria (de los seis hasta los doce años de edad) instituyendo la Educación Pública - en todo el territorio nacional - sobre los principios de laicidad, gratuidad y obligatoriedad.

La formación de docentes, en los países del Río de la Plata, tiene una fuerte tradición normalista, podemos identificar en ella un pensamiento pedagógico claramente positivista pero -a la vez- impregnado de pragmatismo pedagógico tanto a través de las obras de Sarmiento, en Argentina, como la de Varela de 1868 y del corpus de ideas emanadas de la Sociedad de Amigos de la Educación del Pueblo en Uruguay. Así, se articulan, en el siglo XIX estos Sistemas de Educación Nacional Estatal que - más adelante - se nutrirán de las influencias de corrientes pedagógicas innovadoras del siglo XX, hecho particular si lo comparamos con los procesos históricos de la formación docente oficial en otros sistemas educativos de la región (VIERA, 2016).

Esta fuerte tradición normalista, se compensó con la rápida organización de la Formación de docentes en articulación con Educación Primaria y con el fortalecimiento de las "prácticas" o estagios en las carreras de docencia. En Uruguay, este proceso se vio facilitado por el hecho de que Formación Docente y Enseñanza 
Primaria se encontraban dentro del mismo subsistema de Educación, que en su origen se llamó Consejo Nacional de Educación Primaria y Normal (CEPyN); con esto era posible determinar las escuelas de práctica de forma incuestionable. En el caso de formación de maestros para escuelas primarias, los concursos para el cargo de Director de las designadas Escuelas de Práctica, establecían que al Director de Escuelas de Práctica se le sume - a las funciones tradicionales de la gestión escolar - el rol de profesor de Didáctica en la carrera de Magisterio.

Sin embargo, actualmente en Uruguay se presentan nuevos problemas que exige pasar del modelo normalista al universitario, y éste es el desafío actual: caminar hacia una Formación Docente de carácter universitario sin perder la articulación lograda desde el modelo anterior. En términos generales, una de las problemáticas principales con que se enfrentan las políticas públicas en el sector educación - a nivel regional - es cómo mejorar el desempeño de los docentes. Los diagnósticos han coincidido en señalar que "las propuestas tradicionales ya no alcanzan; pero hay también fuertes evidencias de que no es simple determinar cuáles son los cambios adecuados y mucho menos ponerlos en práctica.” (VAILLANT, 2005, p. 39).

Esta preocupación es compartida por diversos investigadores en el campo de las políticas educativas en Argentina y en Latinoamérica en general. Según Fernández Lamarra Y Cópola (2013, p. 3), aún hoy, no se han logrado los niveles de democratización que se anheló desde el principio de la fundación del sistema de educación pública, y esto es más evidente en la Educación Superior y la formación docente.

Si bien en la mayoría de los países latinoamericanos, en las úl-
timas décadas, las políticas educativas han tenido un carácter
prioritario, en el marco de las decisiones, acciones y esfuer-
zos nacionales que se desarrollan, no se han alcanzado nive-
les aceptables de democratización; así la educación superior
en América Latina, muestra fuertes desigualdades en diversos
órdenes tales como la atención de los diversos grupos sociales
y étnicos de población [...] la calidad y pertinencia de la edu-
cación que se ofrece; los niveles de formación de sus docentes.

En Brasil, el proceso de democratización de la Educación y en particular de la formación de los docentes para el Sistema Público de Educación, se ha dado de forma diferente que en Argentina y Uruguay; comenzando por el hecho de que las carreras de graduación de docentes se encuentran dentro de las universidades. Actualmente el Programa Institucional de Bolsas de Iniciação à Docência (PIBID), 
ofrece becas a los alumnos de las carreras docentes para que se dediquen a hacer su práctica en las escuelas públicas con la contrapartida de ejercer luego su profesión en las escuelas de la red pública. El objetivo es fortalecer el vínculo y la articulación entre la Educación Superior (en las carreras de formación de docentes) y las escuelas de los sistemas de educación pública a nivel estadual y municipal.

Esta política muestra la profunda preocupación por provocar el diálogo entre la Formación Docente, dentro de las universidades, y la Educación Básica en los municipios. Una investigación realizada por Sarturi, Possebon e Veleda (2016) muestra que si bien, en estas últimas décadas, Brasil ha intensificado las prerrogativas legales que posibilitan calificar las relaciones entre la educación superior y la educación básica; la realidad actual de las escuelas en Brasil, presentan nuevos desafíos.

[...] os professores são, na maioria das vezes, oriundos de outras regiões da cidade, trabalham em mais escolas, de modo que não conseguem participar efetivamente das atividades da comunidade escolar. Suas ações se restringem à prática em sala de aula, que também não acompanha o projeto da própria escola, pela impossibilidade de participação também em reuniões e outros espaços de trocas, restando-lhe apenas o tempo do recreio para que possa se colocar a par de tudo o que acontece na escola. Tendo a universidade outra dinâmica, ou seja, outro tempo e outro espaço em que as relações acontecem, a professora supervisora exerce um papel fundamental para mediar a comunicação de forma que possa se estabelecer a harmonia, visando superar desafios e atingir objetivos em comum. (SARTURI; POSSEBON; VELEDA, 2016, p. 54).

En esta investigación se revela un sentido nuevo al PIBID, en lo que refiere a la vivencia de la política nacional en los contextos locales. Todo esto llevaría a pensar que se explicita, cada vez más las necesidades de interlocuciones entre la Educación Básica y la formación docente; esto es, el diálogo necesario entre las universidades con otros niveles del Sistema Educativo, en donde el papel de los profesores supervisores, se re-significa y es fundamental en este proceso.

\section{LA INTERNACIONALIZACIÓN DE LA EDUCACIÓN}

A estas cuestiones planteadas anteriormente, hay que agregar los efectos de políticas internacionales determinadas desde organismos multilaterales, y cómo inciden en la interlocución entre la Educación Superior y el nivel Básico de Enseñanza en sus contextos particulares. 
De acuerdo con Martínez Larrechea y Chiancone (2010), la internacionalización sería un fenómeno antropológico en el proceso civilizatorio, y en este sentido, la "internacionalización de la Educación” no sería más que una natural respuesta, a este fenómeno, por parte de los actores que se organizan desde distintos grupos para intercambiar ciencia, tecnología e innovación. Los países desarrollados han sentido la necesidad de organizar espacios universitarios con lógicas internacionales e incluso transnacionales.

En el ámbito universitario, luego de la Declaración de Bolonia, se ha avanzado hacia la lógica de un espacio supranacional de Educación Superior; América Latina - sin embargo - ha mantenido un ritmo mucho más lento en esta tendencia. Aun así, los organismos multilaterales están trabajando activamente en la región. Resulta interesante constatar que la Educación Superior - pensada en términos de globalización - tiene una lógica diferente de la tradicional lógica de los Sistema Educativos Nacionales; pero - aun así - de acuerdo con Hermo (2014), está ocurriendo que aún no se han suprimido las lógicas nacionales al mismo tiempo que están sucediendo fenómenos que trascienden fronteras.

En la Región, parecería, y esto hay que probarlo, que no existe una adecuada vinculación interna de los espacios del Mercosur Educativo con los Ministerios de Educación en cada país. El sector de educación de UNASUR, quiere colocar en la agenda la gestión de la Educación Superior mirando hacia este escenario posible, pero parecería no lograr un cambio profundo en las lógicas tradicionales de la modernidad. Se han realizado propuestas de redes académicas para posibilitar acciones de intercambio desde las universidades y desde los sistemas educativos de los países de la Región; pero existen aún muchos obstáculos a la hora de gestionar estas iniciativas y los tiempos de evolución de este concepto son muy lentos en comparación con Europa. No hay respuestas institucionales a las propuestas de internacionalización de la Educación Superior en el Cono Sur de América. Existen ideas y se presentan proyectos; pero no existe un Plan Estratégico Regional de Educación pensado en clave supranacional. (VIERA; OLIVERA, 2015, p. 92).

Ahora, bien, Latinoamérica tiene el desafío de responder a estos fenómenos de internacionalización de una forma "anticolonialista". Al decir de Tello (2015, p. 93), debemos dejarnos interpelar por la realidad latinoamericana y esto comienza por buscar caminos alternativos legitimados por nuestro propio sentir.

Esto significa que la mirada latinoamericana no consiste sólo en pensar y hacer una lista de los problemas que los países centrales no tienen. Al contrario es asumir la responsabilidad de que los problemas latinoamericanos requieren de un abordaje dife- 
rente, donde la sensibilidad intelectual y la capacidad de estar atentos a los problemas de nuestra región son fundamentales.

Toda América Latina se caracteriza por la diversidad, por tanto, se ha dado un proceso de diversificación de modelos universitarios, que han sido bastante diferentes y contradictorios (FERNÁNDEZ LAMARRA; CÓPPOLA, 2013. Si bien se reconocen tendencias a la innovación universitaria, parecería que muchas instituciones de Educación en este continente tienden a anclarse en un modelo decimonónico.

Para Pérez Lindo (2016), han surgido nuevas tendencias, políticas institucionales, nacionales y regionales que se basan en un nuevo enfoque de la universidad más centrado en la producción y aplicación de conocimientos científicos. Las universidades y las agencias de política científica se abrieron a nuevos programas interdisciplinarios más pragmáticos, se multiplicaron los programas de extensión y de transferencia hacia la sociedad; pero muchas universidades continuaron funcionando como lo que Pérez Lindo (2016) ha dado en llamar “enseñaderos credencialistas" que brindan diplomas que no siempre garantizan la calidad de los graduados.

Es relevante constatar que, si bien entre las comunidades científicas de la Región ha circulado la idea de innovar en investigación, posgrados y cooperación internacional, aun así en los hechos continúa prevaleciendo en algunos países el "tribalismo académico", el pensamiento corporativista, la "feudalización de facultades y de cátedras." (PÉREZ LINDO, 2016). Todo esto parecería estar evidenciando que aún no hubo un cambio profundo de pensamiento en materia de políticas educativas.

El surgimiento de proyectos en varios países latinoamericanos pretenden reforzar la investigación y promover la cooperación internacional; pero en el marco del escenario Regional, estos esfuerzos resultan diferentes a la conformación del Espacio Europeo de Educación Superior. Cada vez más, los organismos internacionales Banco Mundial, Unesco, OCDE, etc. - ejercen una influencia determinante en las políticas educativas de los países subdesarrollados, especialmente en el ámbito de la educación superior.

Algunas voces explican por qué en América Latina, no se quiere seguir el modelo europeo, como lo ha expresado

A diferencia de Bolonia, pensamos en un proceso de integración de la Educación Superior como un proceso de intercambio desde la solidaridad académica. En este sentido nos oponemos a los cánones y fórmulas de "modernización" que ha traído la década de 1990 a las políticas públicas en general y las políti- 
cas educativas en particular. En esta línea, la propia realidad de nuestras universidades se caracterizó por tres componentes claves en los últimos veinte años: a) un fuerte incremento de asistencia de estudiantes a las casas de estudios en términos de masificación; b) mercantilización en términos de performatividad y accountability y c) la búsqueda de la internacionalización institucional, que no necesariamente implica internacionalización del conocimiento como conocimiento público. (TELLO, 2015, p. 82, subrayado nuestro).

En este escenario de internacionalización, algunos investigadores como Martínez Larrechea (2015) opinan que los sudamericanos deberíamos pensar en estrategias que combinen el uso intensivo del conocimiento, la solidaridad social, la sustentabilidad ecológica y el desarrollo, de manera más integrada. Esto nos lleva a la idea de una universidad ligada a un desarrollo inteligente y solidario, por tanto a la idea de repensar la función del conocimiento en el Sur Global.

Por otra parte, las universidades americanas del Sur del planeta - en general - comparten una responsabilidad importante en lo que refiere a los desafíos de la democratización con calidad en sus Sistemas Educativos, especialmente sobre la Educación Básica universal de los países periféricos (MARTÍNEZ LARRECHEA, 2016). La formación de docentes para la Educación básica, en estas regiones, se enfrenta a varios desafíos y tensiones, a modo de ejemplo: la tensión entre lograr una rápida cobertura de profesores para la Enseñanza Básica y - al mismo tiempo elevar las exigencias de las carreras docentes para mejorar la calidad de la formación universitaria de profesores.

\section{LOS ORGANISMOS MULTILATERALES Y LA EDUCACIÓN}

Para Fernandez Lamarra y Albornoz (2014), los agentes que han impulsado la internacionalización de la educación, han sido diversos: las universidades, los ministerios, los programas de cooperación y movilidad promovido por organismos internacionales como la Organización de las Naciones Unidas para la Educación, la Ciencia y la Cultura (Unesco), el banco Mundial (BM), Organización para la Cooperación y Desarrollo Económico (OCDE), el Programa de Naciones para el Desarrollo (PNUD), la Organización de los Estados Americanos (OEA) y Banco Interamericano de Desarrollo (BID); así como diversas Redes y Fundaciones que 
promueven el intercambio estudiantil y docente, entre otras que Fernández Lamarra y Albornoz (2014) mencionan exhaustivamente.

Es de destacar - actualmente - la creación de Redes de Agencias Nacionales de Acreditación ocupadas de los controles de calidad y en búsqueda de estándares internacionales debido - justamente - a las nuevas realidades de la Educación Superior provocadas por el fenómeno de la internacionalización. Ahora bien, dentro de este panorama, resulta llamativo el hecho de que Uruguay no tenga una Agencia Nacional de Acreditación, y es notorio que aún nos falta profundizar más - dentro de la Región - en discusiones acerca de la búsqueda de convergencias, defendidas por algunas voces y cuestionadas por otras.

De acuerdo con Fernández Lamarra (2010), a pesar de los avances, aún existen profundas divergencias en las políticas educativas en América Latina. Para este investigador, habría que avanzar hacia una verdadera integración regional de las naciones latinoamericanas, como lo viene haciendo la Universidad Nacional Tres de Febrero (UNTREF) de Argentina en la constitución de la red Red Internacional para la Convergencia de la Educación Superior (RICES), entre otras.

En el mismo sentido, también se están constatando divergencias en lo que refiere a métodos de investigación y paradigmas en la producción de conocimiento en este campo de estudio. Según Diodati y Nosiglia (2007), en las investigaciones sobre las propuestas desarrolladas por los Organismos Multilaterales sobre Educación, se utilizaban métodos tradicionales de análisis comparativo -para detectar recurrencias o contradicciones entre los diferentes documentos- a través del clásico análisis de contenido; pero de acuerdo a estos autores, en 2007 ya se había asomado un cambio de paradigma en la metodología de la educación comparada, que implica adoptar estrategias de análisis crítico de las propuestas de los Organismos Multilaterales a fin de detectar tanto convergencias como divergencias. En una investigación - en curso - (VIERA, 2017), se adopta estas nuevas estrategias de análisis de los documentos con los objetivos de:

a) comparar los discursos de las políticas educativas nacionales y acuerdos bilaterales con las interpretaciones de los actores en territorios;

b) aportar a la reflexión metodológica de las investigaciones sobre Educación, Internacionalización, Ciudadanía e Inclusión, a fin de buscar nuevos paradigmas de estudio sobre la Educación Superior para los ciudadanos en contextos supranacionales. 
La preocupación por el cambio hacia los nuevos paradigmas de investigación en materia de políticas educativas continúan presentes en la agenda académica; esto se evidencia en el hecho de que en muchos congresos, seminarios y otros eventos internacionales, se propongan - entre sus ejes temáticos - las nuevas metodologías de la educación comparada, nuevos discursos, nuevos enfoques epistemológicos. Hay varios ejemplos recientes de esta preocupación, el propio lema elegido para el XVI Congreso Mundial de Educación Comparada en Beijing de 2016: "Dialéctica de la Educación" es, claramente, uno de ellos. Las discusiones metodológicas para este campo disciplinar tienen su origen en las corrientes filosóficas del siglo XX.

Cabe recordar que Apple $(1986,1996)$ ya había propuesto el análisis crítico de las políticas educativas a través de su concepto teórico y metodológico de "análisis relacional" de las políticas, que implica estudiar estos fenómenos desde su complejidad y de forma contextual. Apple ya planteaba - en las décadas del 1980 y 1990 del siglo pasado - que los grupos contradictorios podrían hacer "alianzas hegemónicas amplias" posibilitando hallar sentido dentro de un paraguas ideológico que admita la diversidad. El planteo de Apple se centra en que las políticas educativas incluyen factores culturales, además de los conocidos factores económicos y el papel del Estado entra en la discusión debido - entre otras cosas - a la preocupación por la transformación social que deviene de las teorías críticas neo marxistas del siglo XX.

Desde esta perspectiva, no debería llamarnos la atención el hecho de que las políticas educativas latino-americana sean tan diversas y presentan contradicciones, porque son el resultado de las disputas que ocurren naturalmente en los Estados. La concepción de políticas culturales supera el paradigma de linealidad y verticalidad; son concebidas como un proceso continuo, dinámico que se deben reinterpretar y reconstruir permanentemente en procesos contra-hegemónicos a fin de que los docentes dejen de reproducir el currículum prescripto (APPLE, 1997).

Desde este interés constitutivo del saber - que da lugar a la ciencia crítica - (HABERMAS, 1990), surgen nuevos desafíos para la formación de los profesores y para la producción de conocimiento sobre la formación docente y las políticas educativas. Superar la linealidad en la investigación permite explicar fenómenos sociales complejos, al dar cabida a marcos teóricos contrarios que - con la debida y rigurosa vigilancia epistemológica - pueden resultar complementarios y más adecuados para explicar la realidad política, generalmente ambigua y paradójica. 
Los planteos de Apple, y de otros pensadores críticos, deberían potencializarse en el análisis de políticas internacionales; ya que el investigador crítico está comprometido con la transformación de la realidad; esto es que no se conforma con la descripción de fenómenos desde una racionalidad técnica (HABERMAS, 1990) sino con el interés práctico de colocar en evidencia los conocimientos de elite a través de procesos contra-hegemónicos que posibiliten políticas de transformación de la realidad humana, superando la obsesión por la neutralidad y la objetividad de la ciencia positiva.

\section{CONSIDERACIONES FINALES}

Luego de una construcción del estado del arte sobre el tema - basado en la revisión de la literatura especializada - y a partir de resultados de investigaciones anteriores e plantea la existencia de una tensión entre las demandas y desafíos de la educación básica de cada país con las innovaciones de la Formación Docente instalada dentro de una Educación Superior cada vez más internacionalizada. Teniendo en cuenta que la implementación de pautas educativas regionales provocan una serie de desafíos para los sistemas nacionales y sus instituciones en contexto, se visualizan una serie de interferencias de las políticas públicas educativas internacionales - principalmente de la Región - en las realidades nacionales y locales.

En el presente artículo se ha tratado de difundir los resultados parciales de un estudios comparado - en curso - que evidencia las diferencias - en materia de políticas educativas para la enseñanza básica y la formación de docentes - debido a los distintos procesos históricos que se han dado en los países del cono sur de Latinoamérica, concretamente en Uruguay, Argentina y Brasil.

Ante los cambios paradigmáticos, estaríamos en condiciones de plantear cómo la diversidad puede visualizarse en tanto obstáculo para la integración latinoamericana; pero también puede ser vista como una característica regional que se consolida en oportunidad de innovaciones y producción de nuevos conocimientos desde otras racionalidades y nuevos enfoques de ciencia que superan las lógicas técnicoinstrumentales de tendencia homogeneizante en materia de políticas educativas. Es así que, las interferencias de los organismos multilaterales en las políticas educativas nacionales y contextuales, pueden interpretarse como inculcación ideológica, así como trabas a las identidades y demandas locales; pero deberíamos (re)pensar, de forma complementaria, la posibilidad de hacer retro actuar - cada vez más - las innovaciones 
locales nacionales para que los organismos internacionales puedan ayudar a implementar y mejorar políticas educativas que demandan los contextos; por ejemplo: una mirada en perspectiva transnacional podría facilitar los diálogos necesarios entre las universidades, la formación docente y la educación básica dentro de las naciones.

Habría - entonces - que pensar cómo articular o re/articular estas relaciones regionales-nacionales-sub nacionales, en sus distintos niveles, para buscar acuerdos a partir de sus lógicas particulares de manera tal que se puedan superar las dificultades entre el conocimiento académico producido por la investigación desde las universidades, y la real contribución - que este campo de conocimiento - puede hacer a quienes toman las decisiones en los rumbos pedagógicos y en cuestiones de gestión educativa desde los gobiernos. En este sentido, los acuerdos multilaterales podrían oficiar de garantía.

El desafío también está dado en encontrar - en forma conjunta - un modo de articular o (re)articular acuerdos a partir de lógicas tan diversas, de manera tal que se puedan superar las dificultades entre el conocimiento académico de las redes internacionales, con el conocimiento empírico que tienen los actores sobre sus propios problemas en territorio. Esto implica crear otro modelo de interlocución que, para lograrlo, habrá que abandonar las lógicas lineales y fragmentarias del pensamiento homogeneizador de la modernidad, para atreverse a abordar los problemas en forma conjunta, desde una concepción dialéctica, que permita la convivencia de visiones opuestas y complementarias a la vez.

Este cambio de pensamiento implica abandonar las prácticas sectarias, dejar de negar las contradicciones y evitar la explicación de la realidad a partir de falsas dicotomías. El reto - en tal sentido - es un llamado para que los intelectuales se abran a discusiones propositivas con otros actores de la sociedad - a nivel local, nacional e internacional - en procura de diálogos comprometidos con una real transformación del mundo y con la construcción de nuevas visiones de las políticas educativas para un cambio hacia el pensamiento sistémico en la toma de decisiones. Producir conocimiento a partir de interlocuciones implica una superación de la racionalidad técnica y una avanzada hacia la racionalidad práctica o hacia un enfoque crítico de la ciencia como lo analiza Habermas en su teoría de los intereses constitutivos del saber (VIERA-DUARTE, 2005, 2016).

Los avances de estas interlocuciones, exigen procesos contra-hegemónicos, de los que hablaba para formar a los docentes en un espíritu crítico-emancipatorio 
que les permita dejar de reproducir el currículum impuesto, y asumir una actitud transformadora y comprometida con la realidad. Finalmente, para que esta manida idea deje de ser una mera expresión de deseo; habrá que comprometerse con un profundo cambio en la concepción de la profesión docente y de las carreras de Formación Inicial y continuada de los profesores; nuevos perfiles, nuevos abordajes que procuren resolver el conocido problema de la "relación teoría-práctica" a través de verdaderos diálogos con la enseñanza básica y las investigaciones internacionales, que los prepare para ser partícipes en las políticas educativas formuladas, diseñadas, implementadas y evaluadas - de forma conjunta - por los todos los actores involucrados en estos procesos.

Habrá que tener en cuenta, también, que los cambios reales pasan por continuar implementando innovaciones pedagógicas que estén acompañadas por cambios estructurales y administrativos; y este aspecto no depende solamente de Formación Docente, sino de un proyecto estratégico planificado para todo el sistema educativo en su conjunto.

Concretamente - en lo que refiere a la formación de grado para docentes se percibe como pertinente acrecentar áreas curriculares destinadas a la preparación para la participación en la planificación y gestión de los centros educativos, así como para la comprensión de las políticas públicas en Educación. Como resultados de investigaciones anteriores existe la idea de que la figura del Director de la escuela, y los mandos medios, son claves para facilitar u obstaculizar procesos de participación y gestión democrática de las escuelas.

Luego de las presentes reflexiones - sustentadas por la revisión de los antecedentes y los hallazgos de sucesivas investigaciones - se sugiere cambiar la planificación de la formación inicial de profesores, incorporando la preparación de los noveles docentes para comprender, acompañar y participar activamente en cuestiones de políticas educativas, así como protagonizar la gestión democrática de las escuelas. Esta Formación inicial, debería estar articulada con una sistematizada formación de posgrados para Directores con perfil de liderazgo pedagógico y gestión educativa democrática. A su vez - estos posgrados - deberían estar articulados con ofertas de especializaciones para directores de "escuelas de práctica" con énfasis en la formación pedagógica del profesor novato.

Todo este sistema de formación inicial y de posgrados generaría naturalmente la formación profesional continua de los docentes. Se refuerza la necesidad de un marco común dentro de un verdadero sistema educativo nacional 
que contemple la diversidad y las interlocuciones necesarias para lograr calidad con equidad en una Educación que necesita interactuar con los organismos multilaterales y las redes académicas, producir conocimiento en materia de políticas educativas y realizar aportes adecuados a las demandas locales, nacionales e internacionales.

\section{REFERENCIAS}

APPLE, M. Ideología y Currículo. Madrid: Akal, 1986.

APPLE, M. Maestros y textos: una economía política de relaciones de clase y sexo en educación. Barcelona: Paidós, 1997.

APPLE, M. Política, cultura y educación. Madrid: Morata, 1996.

CORRALES AYALA, S. La Misión de la Universidad en el Siglo XXI. Razón y Palabra, México, n. 57, jun./jul. 2007. Disponible en: <http://www.razonypalabra. org.mx/anteriores/n57/scorrales.html>. Acceso en: 28 mayo 2016.

DIODATI, M.; NOSIGLIA, C. Organismos multilaterales: un estudio en perspectiva comparada de sus lineamientos y estrategias en materia de educación superior universitaria durante las últimas décadas. In: CONGRESO SAECE, 2., Buenos Aires, 2007. Anales electrónicos... Buenos Aires, 2007. Disponible en: $<$ https://es.scribd. com/document/290996207/organismos-multilaterales>. Acceso en: 14 jul. 2016.

FAZIO, M. Internacionalización del currículum y educación comparada. In: SEMINARIO DE TESIS SOBRE POLÍTICAS DE EDUCACIÓN SUPERIOR EN EL DEPARTAMENTO DE INVESTIGACIONES EDUCATIVAS (DIE), 2015, Cinvestav, Méjico. Anales... Cinvestav, Méjico, 2015.

FAZIO, M. C. Retos y prospectiva de internacionalización en América Latina. Red sobre Internacionalización y Movilidades Académicas y Científicas (RIMAC). UNESCO. IESALC. Venezuela, 2014. Disponible en: < http://iesalc. unesco.org.ve/index.php?option=com_content\&view $=$ article \&id=3545:retos-y-prospectiva-de-la-internacionalizacion-de-la-educacion-superior-en-america-latina\&catid=201\&Itemid=770\&lang=es $>$. Acceso en: 07 oct. 2016.

FERNANDEZ LAMARRA, N.; ALBORNOZ, M. La internacionalización de la Educación Superior y la Ciencia en Argentina In: DIDOU AUPETIT, S.; JARAMILLO DE ESCOBAR, V. (Org.) Internacionalización de la educación Superior y las Ciencias en América Latina. Un Estado del Arte. Panamá: IESALC, 2014. 
FERNANDEZ LAMARRA, N.; CÓPPOLA, N. Desafíos para la construcción del espacio latinoamericano de educación superior, en el marco de las políticas supranacionales. Journal of supranational policies of education, Madrid, n. 1, p. 67-82. jun. 2013.

HABERMAS, J. Conocimiento e interés. Buenos Aires: Taurus, 1990. Original en alemán: Erkenntnis und Interesse, 1968. Surhkamp Verlag. Frankfurt am Main.

HERMO, J. Nuevos desafíos para la gobernanza y los estados nacionales en la globalización. MERCOSUR Y UNASUR. Espacio Abierto Cuaderno Venezolano de Sociología, v. 23, n. 4, 567-585, oct./dic. 2014. Disponible en: <http://www.redalyc. org/pdf/122/12232821001.pdf>. Acceso en: 06 jul. 2017.

IBÁÑEZ, J. El regreso del sujeto. La investigación social de segundo orden. Madrid: Siglo XXI, 1994.

MORIN, E. Introducción al Pensamiento Complejo. Barcelona: Gedisa, 1998.

MARTÍNEZ LARRECHEA, E.; CHIANCONE, A. Políticas educativas en el Conos Sur. Dimensiones críticas de cambio. Magró: Montevideo, 2010.

MARTÍNEZ LARRECHEA, E. La nueva educación comparada en un mundo global: una perspectiva sudamericana. In: ACEVEDO, F.; NOSSAR, K.; VIERA, P. (Org.). Miradas sobre educación y cambio. Rivera-Livramento: IVJBE, 2016. p. 79-87.

PEREZ LINDO, A. La idea de una universidad sudamericana para el desarrollo inteligente y solidario. Conferencia Inaugural Del Instituto Universitario Sudamericano. Montevideo, 2016.

RAMA VITALE, C. La Universidad sin fronteras. Montevideo: Magró, 2015.

RAMA VITALE, C. Políticas y reformas universitarias en América Latina. Montevideo: Magró, 2016.

SARTURI, R.; POSSEBON, C.; VELEDA, N. Políticas públicas educacionais: a experiência do programa de iniciação à docência. In: ACEVEDO, F.; NOSSAR, K.; VIERA, P. (Ed.). Miradas sobre educación y cambio. Rivera-Livramento IV JBE, 2016. p. 57-65.

TELLO, C. Políticas educativas, Educación Superior y proceso de Bolonia en Latinoamérica. Crítica Educativa, São Paulo, n. 1, v. 1, p. 80-97, enero/jun. 2015.

VAILlANT, D. Reformas Educativas y rol docente. PRELAC - Protagonismo docente en el cambio educativo, Santiago, n. 1, p. 38- 51. jul. 2005. 
VARELA, J. P. La educación del Pueblo. Montevideo: CEPyN, 1868.

VIERA, P. Culturas institucionales locales y políticas educativas nacionales. Estudio hermenéutico-fenomenológico en un caso. Análisis y meta- análisis. 2013. Tesis (Doctorado en Educación)-Facultad de Ciencias de la Educación (UDE), Montevideo, 2013.

VIERA, P. La formación docente en Uruguay: entre tradiciones e innovaciones. Revista eletrônica Professare, n. 1, v. 3, p. 6-9. 2014.

VIERA, P. La interdisciplinariedad en la formación docente para educación básica. In: PEDRO, J.; DE SÁ FREIRE, P. Interdisciplinaridade: Universidade e inovação social e tecnológica. Curitiba: CRV, 2016.

VIERA, P.; OLIVERA, R. Internacionalización de la Educación. Una experiencia educativa en zona de frontera: Oportunidades y desafíos. In: PEREIRA DINIS, M.; MOURA DE MELLO, L. (Org.) Cursos binacionais: relatos de uma experiência. Santana do Livramento: Cia do eBook, 2015. p. 87-95. Disponible en: <https://www. researchgate.net/publication/283644603_Cursos_binacionais_relatos_de_uma_experiencia_inovadora>.Acceso en: 06 jul. 2017.

VIERA, P. Rumo a novos formatos de convergência do ensino superior nas regiões de fronteiras no cone sul da américa latina: avanços da pesquisa. In: CONGRESSO IBERO-AMERICANO DE EDUCAÇÃO COMPARADA (CIEC), 2., ENCONTRO INTERNACIONAL DA SOCIEDADE BRASILEIRA DE EDUCAÇÃO COMPARADA (EISBEC), 7., João Pessoa, 2017. Anales electrónicos... João Pessoa, 2017. Diponible en: <http://www.educacaocomparada.com.br/anexoResumo/8fc7b301427 15582fla630f3fa47c2dd.pdf>. Acceso en: 20 jul. 2017.

VIERA, P. Sonancias y Resonancias de un Cambio Educativo. La relación entre Cultura Institucional y Reforma de la Educación. 2005. 137 p. Tesis (Maestría en Educación)-Universidad ORT Montevideo, Uruguay, 2005.

Recebido em: 03 de julho de 2017

Aceito em: 08 de agosto de 2017

Endereço para correspondência: Ituzaingó 667, Rivera, Uruguay; pviera99@gmail. com 